\title{
Challenges in diagnosis in couple and family systems work
}

\begin{abstract}
Choosing to use a formal diagnosis in couples and family counseling can be a treatment conundrum for counselors who believe they must provide a diagnosis. To create a diagnosis for financial reimbursement is a fraudulent and an unethical practice. This article addresses diagnosis in couples and family work and offers strategies to use when not diagnosing.
\end{abstract}

Keyword: Diagnosis; Couples and family counseling; Ethical decision making 